\title{
PROJECTIVE SURFACES OVER A FINITE FIELD
}

\author{
ROGER WIEGAND ${ }^{1}$ AND WILLIAM KRAUTER
}

\begin{abstract}
Let $k$ be the algebraic closure of a finite field, and let $X$ be an irreducible projective surface over $k$. Let $C$ be a curve on $X$, and let $\Omega$ be a finite set of closed points of $X$ meeting each irreducible component of $X$. We prove that there is an irreducible curve on $X$ whose set-theoretic intersection with $\boldsymbol{C}$ is $\Omega$. Using this theorem we characterize $\mathbf{P}_{k}^{2}$ as a topological space, and we show that for any two irreducible plane curves $C, C^{\prime}$ there is a homeomorphism from $\mathbf{P}_{k}^{2}$ onto itself taking $C$ onto $C^{\prime}$.
\end{abstract}

Our ground field will always be the algebraic closure $k$ of a finite field of arbitrary characteristic. The word "surface" will always mean "complete surface over $k$ ". If $C$ is a reduced curve with irreducible components $C_{1}, \ldots, C_{n}$, lying on a surface $X$, we will denote the intersection matrix $\left\langle C_{i} \cdot C_{j}\right\rangle$ simply by $\langle C \cdot C\rangle$. We will work exclusively with closed points, and the symbol " $\cap$ " will always denote set-theoretic intersection. Similarly, if $\alpha: X_{1} \rightarrow X_{2}$ is a map of surfaces and $A_{i}$ is a subset of $X_{i}$, then $\alpha\left(A_{1}\right)$ and $\alpha^{-1}\left(A_{2}\right)$ denote the set-theoretic image and inverse image, respectively.

Our main theorem follows from a theorem of $\mathbf{M}$. Artin [A], which states that a connected curve $C$ on a nonsingular surface $X$ can be contracted, provided $\langle C \cdot C\rangle$ is negative-definite. In other words, there is a map $\alpha$ from $X$ onto a surface $Y$, such that $\alpha(C)$ is a single point and $\alpha$ is an isomorphism over $Y-\alpha(C)$. We are very grateful to Michael Artin for suggesting this approach. Our original proof proved less.

We will need the following simple lemma from linear algebra:

LEMMA 1. Let $A$ be a symmetric negative-definite $n \times n$ matrix with real entries, and let $b \in \mathbf{R}^{n}$. Then for all $N \ll 0$ the matrix

$$
B=\left[\begin{array}{c|c}
A & b^{t} \\
\hline b & N
\end{array}\right]
$$

is negative-definite.

Proof. Let $\delta$ be the maximum value of $y A y^{t}$ on the sphere $\|y\|=1$, and take $N<\|b\|^{2} / \delta$. If $x=\left(y_{1}, \ldots, y_{n}, z\right)$ is nonzero, we have

$$
\begin{aligned}
x B x^{t} & =y A y^{t}+2 z b y^{t}+N z^{2} \leqslant \delta\|y\|^{2}+2|z|\|b\|\|y\|+N z^{2} \\
& =\delta(\|y\|+|z|\|b\| / \delta)^{2}+\left(N-\|b\|^{2} / \delta\right)|z|^{2}<0 .
\end{aligned}
$$

Received by the editors December 4, 1980 and, in revised form, January 8, 1981.

1980 Mathematics Subject Classification. Primary 14C20.

'This author's research was partially supported by a grant from the National Science Foundation. 
In order to keep the notation simple it is helpful to state our theorem first for nonsingular surfaces. Here the result is a little sharper.

LEMMA 2. Let $C$ and $E$ be curves on the nonsingular surface $X$, and let $\Omega$ and $\Psi$ be finite subsets of $C$ and $X-(C \cup E)$, respectively. Assume $\langle E \cdot E\rangle$ is negativedefinite, $\Omega \cap E=\varnothing$, and $\Omega$ meets each irreducible component of $C$. Then there is an irreducible curve $D$ on $X$ such that $D \cap C=\Omega$ and $D \cap(E \cup \Psi)=\varnothing$.

Proof. We will blow up points of $\Omega$ repeatedly until the proper transform of $E \cup C$ has negative-definite intersection matrix. To do this, we first blow up $\Omega$, and let $X^{\prime} \stackrel{\alpha}{\rightarrow} X$ be the resulting transformation. If $\alpha^{-1}(C)$ has multiplicity $>3$ at some point $P$ of $\alpha^{-1}(\Omega)$, blow up $P$. This decreases the arithmetic genus of $\alpha^{-1}(C)$, by the proof of [H, V, Theorem 3.9]. Since the arithmetic genus is bounded below by $1-(\#$ of connected components of $C)$, we eventually get a transformation, still denoted by $\alpha: X^{\prime} \rightarrow X$, such that (a) $\alpha^{-1}(x)$ is a curve for each $x \in \Omega$, (b) $\alpha$ is an isomorphism over $X-\Omega$, and (c) $\alpha^{-1}(C)$ has multiplicity 2 at each point of $\alpha^{-1}(\Omega)$.

Let $C_{1}, \ldots, C_{n}$ be the irreducible components of $C$, and let $C_{i}^{\prime}$ be the proper transform of $C_{i}$ on $X^{\prime}$. Choose, for each $i$, a point $P_{i} \in C_{i}^{\prime} \cap \alpha^{-1}(\Omega)$. By (a), (b) and (c), $C_{i}$ is nonsingular at $P_{i}$, and $P_{i} \notin C_{j}^{\prime}$ if $i \neq j$. Also, $P_{i}$ is not on $E^{\prime}$, the proper transform of $E$. Therefore, when we blow up $P_{i}$ and replace $E^{\prime} \cup C^{\prime}$ by its proper transform, the only entry in $\left\langle\left(E^{\prime} \cup C^{\prime}\right) \cdot\left(E^{\prime} \cup C^{\prime}\right)\right\rangle$ that changes is $C_{i}^{\prime} \cdot C_{i}^{\prime}$, which drops by 1 . Since $\left\langle E^{\prime} \cdot E^{\prime}\right\rangle=\langle E \cdot E\rangle$, Lemma 1 says we can blow up $P_{1}$ sufficiently often, and then blow up $P_{2}, \ldots, P_{n}$ repeatedly, so that the proper transform $\tilde{E} \cup \tilde{C}$ on our new surface $\tilde{X}$ has negative-definite intersection matrix.

Let $\beta$ be the composite map $\tilde{X} \rightarrow X^{\prime} \rightarrow X$. Then $\beta$ is still an isomorphism over $X-\Omega$, and $\beta^{-1}(x)$ is a curve for every $x \in \Omega$. By [A, Theorem 2.9], we can contract the connected components of $\tilde{E} \cup \tilde{C}$ successively to obtain a normal surface $Y$ and a map $\gamma: \tilde{X} \rightarrow Y$ such that $\gamma(\tilde{E} \cup \tilde{C})$ is a finite set and $\gamma$ is an isomorphism off $\tilde{E} \cup \tilde{C}$. By [A, Corollary 2.11], $Y$ is projective, and a suitable hyperplane section will provide an irreducible curve $H$ avoiding the finite set $\gamma(\tilde{E} \cup \tilde{C}) \cup \gamma \beta^{-1}(\Psi)$. (Since most treatments of Bertini's theorem require $Y$ to be nonsingular, we refer to [S, Theorem 1.1], which says $H$ is locally irreducible, and [H, III, Corollary 7.9], which says $H$ is connected.) Now $H$ meets each of the curves $\gamma \beta^{-1}(x), x \in \Omega$. Letting $\tilde{H}$ be the proper transform of $H$ on $\tilde{X}$, we see that $D=\beta(H)$ is the required curve on $X$.

MAIN THEOREM. Let $C$ be a curve on a surface $X$, and let $\Omega$ and $\Psi$ be finite subsets of $C$ and $X-C$, respectively. Suppose $\Omega$ meets each irreducible component of $C$. Then there is an irreducible curve $D$ on $X$ such that $D \cap C=\Omega$ and $D \cap \Psi=\varnothing$.

Proof. Suppose we can prove the theorem for normal surfaces. Let $X^{\prime} \stackrel{\pi}{\rightarrow} X$ be the normalization, and write $\pi^{-1}(C)=C^{\prime} \cup \Delta$, where $C^{\prime}$ is a curve and $\Delta$ is a finite set disjoint from $C^{\prime}$. Choose a finite set $\Omega^{\prime} \subset C^{\prime}$, meeting each irreducible component of $C^{\prime}$ and mapping onto $\Omega$ via $\pi$. Apply the theorem to $X^{\prime}, C^{\prime}, \Omega^{\prime}$ and 
$\Psi^{\prime}=\pi^{-1}(\Psi) \cup \Delta$ to get an irreducible curve $D^{\prime}$ on $X^{\prime}$ such that $D^{\prime} \cap C^{\prime}=\Omega^{\prime}$ and $D^{\prime} \cap \Psi^{\prime}=\varnothing$. Setting $D=\pi\left(D^{\prime}\right)$, we see that the theorem holds for $X$.

Changing notation, we now assume $X$ is normal. Let $Q_{1}, \ldots, Q_{s}$ be the singular points of $X$, numbered so that $Q_{1}, \ldots, Q_{r} \notin \Omega$ and $Q_{r+1}, \ldots, Q_{s} \in \Omega$. By repeatedly blowing up points lying over $Q_{1}$ and normalizing, we get a normal surface $X_{1}$ and a map $\alpha_{1}: X_{1} \rightarrow X$, such that $X_{1}$ is nonsingular at each point of $\alpha_{1}^{-1}\left(Q_{1}\right)$ and $\alpha_{1}$ is an isomorphism over $X-\left\{Q_{1}\right\}$. Continuing, let $\alpha_{i}: X_{i} \rightarrow X_{i-1}$ be a similar desingularization of $Q_{i}, 2<i<s$, and let $\alpha: \tilde{X} \rightarrow X$ be the composition $\alpha_{1} \cdots \alpha_{s}$. Then $\tilde{X}$ is a nonsingular surface, and $\alpha$ is an isomorphism over $X-\left\{Q_{1}, \ldots, Q_{s}\right\}$.

Let $E_{i}=\alpha^{-1}\left(Q_{i}\right), 1<i<r$, and let $E=E_{1} \cup \cdots \cup E_{r}$. By Zariski's main theorem [H, III, Corollary 11.4], each $E_{i}$ is a connected curve. When viewed in $X_{i}$, $E_{i}$ is contractible, and hence has negative-definite intersection matrix [M]. But $\left\langle E_{i} \cdot E_{i}\right\rangle$ is unchanged by $\alpha_{i+1}, \ldots, \alpha_{s}$; and we conclude that $\langle E \cdot E\rangle$ is negativedefinite.

Now let $\tilde{C}$ be the proper transform of $C$ on $\tilde{X}$, and let

$$
\tilde{\Psi}=\alpha^{-1}(\{\text { nonsingular points of } X \text { in } \Psi\}) \text {. }
$$

Choose a finite set $\tilde{\Omega} \subset \tilde{C}$ meeting each component of $\tilde{C}$ and mapping onto $\Omega$ via $\alpha$. By Lemma 2 there is an irreducible curve $\tilde{D}$ on $\tilde{X}$ such that $\tilde{D} \cap \tilde{C}=\tilde{\Omega}$ and $\tilde{D} \cap(E \cup \tilde{\Psi})=\varnothing$. Finally, let $D=\alpha(\tilde{D})$. One checks easily that $D \cap C=\Omega$ and $D \cap \Psi=\varnothing$.

Using the Main Theorem we will classify, up to homeomorphism, the open subsets of $\mathbf{P}_{k}^{2}$. The analogous statements in terms of the topology on the whole scheme, and in terms of the partially ordered set of irreducible closed subsets, are left to the reader. To simplify the statement, we define a "curve" on a topological space $V$ to be a nonempty closed subset of $V$, each of whose irreducible components has dimension 1.

COROLlary 1. Let $k$ be the algebraic closure of a finite field. The space $V$ of closed points of $\mathbf{P}_{k}^{2}$ satisfies the following axioms:

(A1) $V$ is $T_{1}$, Noetherian and irreducible, $\operatorname{dim} V=2$, and every point of $V$ lies on some curve.

(A2) $V$ contains only countably many curves.

(A3) If $C$ is a curve on $V$ and $\Omega$ is a finite subset of $C$ meeting each component of $C$, there is an irreducible curve $D$ on $V$ such that $D \cap C=\Omega$.

(A4) Every curve on $V$ is connected.

Moreover, any two topological spaces satisfying (A1)-(A4) are homeomorphic.

Axioms (A1), (A2) and (A4) are clear, and (A3) comes from the Main Theorem. Since any two irreducible curves are homeomorphic, the last statement is an immediate consequence of the following stronger result:

Proposition. Let $V$ and $V^{\prime}$ be topological spaces satisfying (A1)-(A4), and suppose $\theta$ is a homeomorphism from some curve $C$ on $V$ onto a curve $C^{\prime}$ on $V^{\prime}$. Then $\theta$ extends to a homeomorphism from $V$ onto $V^{\prime}$. 
Proof. Let $\mathcal{S}$, respectively, $\mathcal{S}^{\prime}$, be the set of irreducible curves in $V$, respectively, $V^{\prime}$. For convenience, we give each set $\delta, \delta^{\prime}$ a well-ordering of type $\omega$. Our homeomorphism $\boldsymbol{\theta}$ induces a bijection from the finite set $\mathcal{T}$ of irreducible components of $C$ onto the set $\mathcal{T}^{\prime}$ of irreducible components of $C^{\prime}$. Let $D$ be the first element of $\mathcal{S}-\mathcal{T}$, and note that $C \cap D$ is a finite set meeting each component of $C$. By (A3) there is some $D^{\prime} \in \mathcal{S}^{\prime}-\mathcal{T}^{\prime}$ such that $C^{\prime} \cap D^{\prime}=\theta(C \cap D)$. We extend $\theta$ to a homeomorphism $\phi$ from $C \cup D$ onto $C^{\prime} \cup D^{\prime}$ by using an arbitrary bijection between $D-C$ and $D^{\prime}-C^{\prime}$. Now let $E^{\prime}$ be the first element of $\mathcal{S}^{\prime}-\left(\mathcal{T}^{\prime} \cup\left\{D^{\prime}\right\}\right)$, and repeat the process, extending $\phi^{-1}$ to a homeomorphism from $C^{\prime} \cup D^{\prime} \cup E^{\prime}$ onto $C \cup D \cup E$, where $E$ is a suitable member of $\mathcal{S}-(\mathcal{T}$ $\cup\{D\})$. After countably many steps of this sort, we get a bijection $\psi: V \rightarrow V^{\prime}$ inducing a bijection from $\mathcal{S}$ onto $\mathcal{S}^{\prime}$. Clearly $\psi$ is a homeomorphism, since proper closed sets are finite unions of points and curves.

COROLlary 2. Let $k$ be the algebraic closure of a finite field, and let $U$ be $a$ nonempty proper open subset of $\mathbf{P}_{k}^{2}$. Then $U$ is homeomorphic to either $\mathbf{P}_{k}^{2}-\{$ point $\}$ or $\mathbf{A}_{k}^{2}$.

Proof. If the complement of $U$ contains a closed curve in $\mathbf{P}_{k}^{2}$ then $U$ is an open subset of an irreducible affine surface over $k$. By [W, Theorem 6 and Corollary 7], $U$ is homeomorphic to $\mathbf{A}_{k}^{2}$. To complete the proof, we need to show that $\mathbf{P}_{k}^{2}-\{P\}$ is homeomorphic to $\mathbf{P}_{k}^{2}-\left\{P_{1}^{\prime}, \ldots, P_{r}^{\prime}\right\}$ whenever $P, P_{1}^{\prime}, \ldots, P_{r}^{\prime}$ are distinct points of $\mathbf{P}_{k}^{2}$ and $r \geqslant 1$.

We imitate the proof above, always working with curves in $\mathbf{P}_{k}^{2}$. Let $X=\mathbf{P}_{k}^{2}-$ $\{P\}, X^{\prime}=\mathbf{P}_{k}^{2}-\left\{P_{1}^{\prime}, \ldots, P_{r}^{\prime}\right\}$, and let $\mathcal{S}=\mathcal{S}^{\prime}$ be the set of irreducible curves in $\mathbf{P}_{k}^{2}$, well-ordered as in the proof of the Proposition. Suppose $\mathcal{T}$ and $\mathcal{T}^{\prime}$ are finite subsets of $\mathcal{S}$ and $\mathcal{S}^{\prime}$, and $\theta$ is a homeomorphism from $(\cup \mathcal{T}) \cap X$ onto $\left(\cup \mathcal{J}^{\prime}\right) \cap$ $X^{\prime}$ satisfying the extra condition: (*) For each $T \in \mathcal{T}$ we have $P \in T \Leftrightarrow$ some $P_{i}^{\prime} \in \theta(T-\{P\})^{-}$. One can use the Main Theorem to extend the domain of either $\boldsymbol{\theta}$ or $\boldsymbol{\theta}^{-1}$ to include the first curve $\delta-\mathcal{T}$ or $\mathcal{S}^{\prime}-\mathcal{T}^{\prime}$. Moreover, this can be done in such a way that the extended homeomorphism still satisfies (*), so the process can be continued indefinitely.

We remark that $\mathbf{P}_{k}^{2}, \mathbf{P}_{k}^{2}-\{P\}$, and $\mathbf{A}_{k}^{2}$ are all pairwise nonhomeomorphic. To see this, note that $\mathbf{P}_{k}^{2}-\{P\}$ and $\mathbf{A}_{k}^{2}$ each contain disconnected curves, so these spaces are not homeomorphic to $\mathbf{P}_{k}^{2}$. Also, for each curve in $\mathbf{A}_{k}^{2}$, say $f=0$, there is another curve $(f=1)$ disjoint from it; whereas $\mathbf{P}_{k}^{2}-\{P\}$ has lots of curves that meet every other curve. (Take any curve in $\mathbf{P}_{k}^{2}$ not passing through $P$.)

Finally, we observe that $X=\mathbf{P}_{k}^{2}-\{P\}$ and $X^{\prime}=\mathbf{P}_{k}^{2}-\left\{P_{1}^{\prime}, P_{2}^{\prime}\right\}$ are not isomorphic. For, if $C$ is any disconnected curve in $\mathbf{P}_{k}^{2}-\{P\}$, then $X-C$ is affine. On the other hand, let $D$ be the union of two distinct lines passing through $P_{1}^{\prime}$ and avoiding $P_{2}^{\prime}$, and let $C^{\prime}=D-\left\{P_{1}^{\prime}\right\}$. Then $X^{\prime}-C^{\prime}=\mathbf{P}^{2}-\left(D \cup\left\{P_{2}^{\prime}\right\}\right)$ is not affine, since the map

$$
A\left(\mathbf{P}^{2}-D\right)=\theta\left(\mathbf{P}^{2}-D\right) \rightarrow \theta\left(\mathbf{P}^{2}-\left(D \cup\left\{P_{2}^{\prime}\right\}\right)\right)
$$

is an isomorphism. (See [H, I], particularly Example 3.20 and Proposition 3.5.) 


\section{REFERENCES}

[A] M. Artin, Some numerical criteria for contractability of curves on algebraic surfaces, Amer. J. Math. 84 (1962), 485-496.

[H] R. Hartshorne, Algebraic geometry, Graduate Texts in Math., vol. 52, Springer-Verlag, New York, 1977.

[M] D. Mumford, The topology of normal singularities of an algebraic surface and a criterion for simplicity, Inst. Hautes Etudes Sci. Publ. Math. 9 (1961), 5-22.

[S] R. G. Swan, A cancellation theorem for projective modules in the metastable range, Invent. Math. 27 (1974), 23-43.

[W] R. Wiegand, Homeomorphisms of affine surfaces over a finite field, J. London Math. Soc. 18 (1978), 28-32.

Department of Mathematics and Statistics, Universtty of Nebraska, Lincoln, Nebraska 68588 